\title{
Novel Biodegradable Polymer Tethered Platinum (II) for Photoacoustic Imaging
}

\author{
Kien Voon Kong ${ }^{1 \#, ~ L u n-D e ~ L i a o ~}{ }^{2 \#}$, Douglas Goh ${ }^{1}$, Nitish V Thakor ${ }^{2,3 *}$ and Malini Olivo ${ }^{1,4 *}$
}

${ }^{1}$ Singapore Bioimaging Consortium, Agency for Science, Technology, and Research (A*STAR), 11 Biopolis Way, \#02-02 Helios, Singapore 138667, Singapore ${ }^{2}$ Singapore Institute for Neurotechnology (SINAPSE), National University of Singapore, 28 Medical Drive, \#05-COR, Singapore 117456, Singapore

${ }^{3}$ Department of Biomedical Engineering, Johns Hopkins University, Traylor 701/720 Rutland Ave, Baltimore, 21205, USA

${ }^{4}$ School of Physics, National University of Ireland, University Road, Galway, Ireland

\#These authors contributed equally to this work

\begin{abstract}
Photoacoustic microscopy (PAM) is an emerging imaging diagnostic technique for various diseases. Coupled with contrast agents, photoacoustic (PA) imaging yields additional information to facilitate an accurate diagnosis. While many organic-based contrast agents, notably those of cyanine dyes, nanoparticles, polyhydroxy-fullerene and carbon nanotubes, have become available, the use of transition metal as contrast agent is scant. Here, for the first time, we report a platinum II-based biodegradable polymer for PA imaging that is capable of effective cellular internalization with very low cytotoxicity. The experiment results show great promise as a novel photoacoustic contrast agent as its detectable PA signal was observed both in cell imaging and in vivo rat cerebral vascular imaging via designed PAM. This work exemplifies the incorporation of transition metal complex with polymeric nanoparticles, further expanding the field of the ability of PA imaging.
\end{abstract}

Keywords: Transition metal; Platinum; Biodegradable polymer; Contrast agent; Photoacoustic imaging

\section{Introduction}

Fluorescence imaging, positron emission tomography (PET), micro-computed tomography ( $\mathrm{m}-\mathrm{CT}$ ) and magnetic resonance imaging (MRI) are the frequently used imaging techniques currently employed in biological studies. In recent years, photoacoustic (PA) imaging has garnered immense attention [1]. PA imaging is a powerful technique that exclusively combines optical and ultrasound imaging where localized heating and increased kinetic energy are generated by adsorbed light energy [2,3]. This technique can be coupled with exogenous contrast agents to obtain more information to facilitate accurate diagnoses [46 . There is vast literature on the use of cyanine dyes, carbon nanotubes and nanoparticles as PA contrast agents. However, the use of transition metal complexes as a PA contrast agent is relatively rare $[7,8]$. This could be attributed partly to their complicated chemistry. On top of that, some transition metal complexes are required to be handled with care due to their air- and moisture-sensitivity and toxicity issues. Nevertheless, there are also many classes of transition metal complexes that are sufficiently stable in air and water, and employable in biological systems $[9,10]$. In fact, some of them have been successfully translated to clinical uses. The use of gadolinium and cisplatin in MRI and chemotherapy, respectively, are two instances that embody the great potential and versatility of transition metal complexes [11,12].

Cisplatin is heralded as one of the most effective chemotherapy agents approved by the Food and Drug Administration since thirty years ago [13-18]. The successful clinical application of cisplatin has been the main impetus for the evolution of platinum complexes and platinum-based delivery system [19-21]. However, the use of platinum complex as a contrast agent appears to be scant. Moreover, there is no report of platinum-based PA agents. This prompted us to investigate the feasibility of using platinum complex for potential applications in diagnostics.

Poly(aminomethylpiperidine-bis(acryloyl)cystamine)(poly(AMPD$\mathrm{BAC})$ ) is a biodegradable poly(amidoamine) that contains multiple reduction-sensitive disulfide bonds, and secondary and tertiary amines in the backbone through Michael-addition polymerization. It offers, in particular, the advantages of easy tunability of their topology, and the availability of multiple sites for the attachment of various ligands as property modifiers such as target-specific ligands and poly(ethylene)glycol stealth layer [22-26]. Given its numerous disulfide bonds, poly(AMBD-BAC) offers enhanced biodegradability that can potentially minimize the long term bodily accumulation of contrast agents after PA studies [27]. The high density of amino groups confers significant buffering capacity over a wide $\mathrm{pH}$ range, giving rise to the "proton sponge effect" that is important for intracellular delivery [2833].

Herein, we present a novel platinum II-based biodegradable nanoparticle that shows significant promise as a PA contrast agent and demonstrate its performance in improving sensitivity for in vivo brain vascular imaging in rat. To the best of our knowledge, this is the first reported preparation of PA contrast agents using biodegradable polymer. The successful demonstration of PA imaging with biodegradable polymer will certainly benefit current delivery processes of PA agents. Also, we believe that this work illustrates the applicability of using polymeric nanoparticle functionalized with other transition metals in the development of PA contrast agents.

*Corresponding authors: Malini Olivo, Singapore Bioimaging Consortium, Agency for Science, Technology, and Research (A*STAR), 11 Biopolis Way, \#02-02 Helios, Singapore 138667, Singapore, Tel: (65) 6478 8752; Fax: (65) 64789957 E-mail: Malini_Olivo@sbic.a-star.edu.sg

Nitish V Thakor, Singapore Institute for Neurotechnology (SINAPSE), Nationa University of Singapore, 28 Medical Drive, \#05-COR, Singapore 117456, Singapore, E-mail: sinapsedirector@gmail.com

Received July 31, 2014; Accepted August 27, 2014; Published August 30, 2014

Citation: Kong KV, Liao LD, Goh D, Thakor NV, Olivo M (2014) Novel Biodegradable Polymer Tethered Platinum (II) for Photoacoustic Imaging. J Nanomed Nanotechnol 5: 223. doi: 10.4172/2157-7439.1000223

Copyright: (C) 2014 Kong KV, et al. This is an open-access article distributed under the terms of the Creative Commons Attribution License, which permits unrestricted use, distribution, and reproduction in any medium, provided the original author and source are credited. 


\section{Materials and Methods}

\section{Characterization}

${ }^{1} \mathrm{H}$ NMR spectra were recorded on a Bruker DRX-400 NMR spectrometer; chemical shifts reported were referenced against the residual proton signals of the solvents. Molecular weight was determined from gel permeation chromatography (GPC) implemented on a Waters 2690 apparatus with Water Ultrahydrogel 250 and 200 columns, a Waters 410 refractive index detector, and a miniDAWN light scattering detector (Wyatt Technology) using $0.2 \mathrm{M}$ acetic acid/ $0.2 \mathrm{M}$ sodium acetate as eluent at a flow rate of $0.75 \mathrm{ml} / \mathrm{min}$. A Brookhaven BI-9000AT Digital Autocorrelator was used for dynamic light scattering (DLS) measurements with $90^{\circ}$ scattering angle laser light of wavelength 632.8 $\mathrm{nm}$. Isocratic reverse phase high performance liquid chromatography (HPLC) was implemented on the Waters 2695 Separation Module with a reverse phase SymmetryShield Column (pore size $5 \mu \mathrm{m}, 150$ $\mathrm{mm} \times 4.6 \mathrm{~mm}$ i.d.) and a Waters 2996 PDA detector with Millennium processing software version 3.2. A mixture solvent of acetonitrile-water $(50: 50, \mathrm{v} / \mathrm{v})$ was used as mobile phase at a flow rate of $1 \mathrm{ml} / \mathrm{min}$ at $25^{\circ} \mathrm{C}$, and the wavelength of the UV detector was set at $227 \mathrm{~nm}$. Transmission electron microscopy (TEM) images were obtained on a Philips CM300 FEGTEM instrument at $300 \mathrm{kV}$. The samples were prepared by dipping holey copper meshes covered with carbon into an aqueous solution of samples followed by drying in air.

\section{Materials}

4-aminomethyl piperidine (AMPD, Alfa Aesar), N,N`-bis(acryloyl) cystamine (BAC, polysciences), paclitaxel (PTX, Yunnan Hande BioTech Co. Ltd, China), dithiothreitol (DTT, Sigm-Aldrich), 4-nitrophenyl chloroformate (Fluka), cisplatin (Sigma), diethylenetriaminepentaacetic acid dianhydride (Sigma) and monomethyl poly(ethylene glycol) (Mw: 2000, Sigma-Aldrich) were from commercial sources and used without further purification. All other starting materials were purchased from Aldrich. MDA-MB-231 cell lines were purchased from the American Type Culture Collection (ATCC). MDA-MB-231 cells were grown in culture flasks with Dulbecco's Modified Engle Medium (DMEM, Invitrogen) containing $10 \%$ fetal bovine serum (FBS, Invitrogen), $1 \%$ L-glutamate (GIBCO Laboratories) and $1 \%$ penicillin-streptomycin (GIBCO Laboratories) at $37^{\circ} \mathrm{C}$ in a $5 \% \mathrm{CO}_{2}$ incubator. Phosphate buffered saline (PBS) was purchased from 1st BASE. The cytotoxicity assay was performed with 3-(4,5-Dimethylthiazol-2-yl)-2,5Diphenyltetrazolium Bromide (MTT, Duchefa Biochemie).

\section{Synthesis of linear polymer poly(AMPD-BAC)}

The polymer was synthesized by Michael-addition polymerization. BAC (6.14 g, $25 \mathrm{mmol}$ ) was dissolved in $40 \mathrm{ml}$ dry methanol at room temperature. AMPD $(2.88 \mathrm{~g}, 25 \mathrm{mmol})$ was added to the solution while stirring. The mixture was stirred at room temperature for 30 days. The resulted solution was dialyzed against methanol for five times to remove unreacted monomers. Yield $=6.80 \mathrm{~g}(71 \%) .{ }^{1} \mathrm{H}$ NMR (CH3OD): $\delta 1.24$ (m, 2H, CH2), 1.52 (bs, 1H, CH), 1.75 (d, 2H, $\mathrm{CH}_{2}$ ), 2.03 (t, 4H, $\mathrm{CH}_{2}$ ), $2.38\left(\mathrm{t}, 4 \mathrm{H}, \mathrm{CH}_{2}\right), 2.49\left(\mathrm{~d}, 2 \mathrm{H}, \mathrm{CH}_{2}\right), 2.65\left(\mathrm{t}, 2 \mathrm{H}, \mathrm{CH}_{2}\right), 2.83\left(\mathrm{t}, 4 \mathrm{H}, \mathrm{CH}_{2}\right)$, $2.95\left(\mathrm{~d}, 2 \mathrm{H}, \mathrm{CH}_{2}\right), 3.49\left(\mathrm{t}, 4 \mathrm{H}, \mathrm{CH}_{2}\right)$.

\section{Synthesis of poly(AMPD-BAC)-g-PEG}

To poly(AMPD-BAC) $(6.0 \mathrm{~g}, 16.0 \mathrm{mmol})$ in $60 \mathrm{ml}$ dry dimethylsulfone was added PEG 4-nitrophenyl carbonate $(5.83 \mathrm{~g}, 2.6$ $\mathrm{mmol}$ ). The mixture was stirred at room temperature for five days. The resulted solution was dialyzed against methanol for 4 times to remove unreacted PEG. The solvent was removed under reduced pressure to afford poly(AMPD-BAC)-g-PEG as a water soluble white solid. Yield $=7.40 \mathrm{~g}(73 \%) .{ }^{1} \mathrm{H}$ NMR $(\mathrm{CH} 3 \mathrm{OD}): \delta 1.24\left(\mathrm{~m}, 2 \mathrm{H}, \mathrm{CH}_{2}\right), 1.52(\mathrm{bs}, 1 \mathrm{H}$, $\mathrm{CH}), 1.75\left(\mathrm{~d}, 2 \mathrm{H}, \mathrm{CH}_{2}\right), 2.03\left(\mathrm{t}, 4 \mathrm{H}, \mathrm{CH}_{2}\right), 2.38\left(\mathrm{t}, 4 \mathrm{H}, \mathrm{CH}_{2}\right), 2.49(\mathrm{~d}, 2 \mathrm{H}$, $\left.\mathrm{CH}_{2}\right), 2.65\left(\mathrm{t}, 2 \mathrm{H}, \mathrm{CH}_{2}\right), 2.83\left(\mathrm{t}, 4 \mathrm{H}, \mathrm{CH}_{2}\right), 2.95\left(\mathrm{~d}, 2 \mathrm{H}, \mathrm{CH}_{2}\right), 3.49(\mathrm{t}, 4 \mathrm{H}$, $\mathrm{CH}_{2}$ ), 3.63 (s, b, $\mathrm{OCH}_{2} \mathrm{CH}_{2}$ ), 4.14 (bs, $2 \mathrm{H}, \mathrm{CH}_{2}$ ).

\section{Synthesis of poly(AMPD-BAC)-g-PEG-DTPA}

To a poly(AMPD-BAC)-g-PEG $(2.0 \mathrm{~g}, 0.21 \mathrm{mmol})$ in $30 \mathrm{ml}$ dry DMSO was added diethylenetriaminepentaacetic acid dianhydride $(0.74 \mathrm{~g}, 2.1 \mathrm{mmol})$ and distilled triethylamine $(0.3 \mathrm{ml}, 2.1 \mathrm{mmol})$. After the mixture was stirred for $24 \mathrm{~h}$ under argon at room temperature, the resulted solution was dialyzed against water for five times and then freeze dried to afford poly(AMPD-BAC)-g-PEG-DTPA a white solid. Yield $=1.0 \mathrm{~g}(50 \%) .{ }^{1} \mathrm{H}$ NMR $\left(\mathrm{CDCl}_{3}\right): \delta 1.24\left(\mathrm{~m}, 2 \mathrm{H}, \mathrm{CH}_{2}\right), 1.52(\mathrm{bs}$, $1 \mathrm{H}, \mathrm{CH}), 1.75\left(\mathrm{~d}, 2 \mathrm{H}, \mathrm{CH}_{2}\right), 2.03\left(\mathrm{t}, 4 \mathrm{H}, \mathrm{CH}_{2}\right), 2.38\left(\mathrm{t}, 4 \mathrm{H}, \mathrm{CH}_{2}\right), 2.49(\mathrm{~d}$, $\left.2 \mathrm{H}, \mathrm{CH}_{2}\right), 2.65\left(\mathrm{t}, 2 \mathrm{H}, \mathrm{CH}_{2}\right), 2.83\left(\mathrm{t}, 4 \mathrm{H}, \mathrm{CH}_{2}\right), 2.95\left(\mathrm{~d}, 2 \mathrm{H}, \mathrm{CH}_{2}\right), 3.49(\mathrm{t}$, $\left.4 \mathrm{H}, \mathrm{CH}_{2}\right), 3.51$ (s, DTPA), 3.78 (s, DTPA), 3.83(s, DTPA), 3.96 (s, 2H, $\mathrm{CH}_{2}$ DTPA), 3.63 (s, b, $\mathrm{OCH}_{2} \mathrm{C}_{\mathrm{H} 2}$ ), 4.14 (bs, $2 \mathrm{H}, \mathrm{CH}_{2}$ ).

\section{Preparation of cisplatin incorporated micelles}

Poly(AMPD-BAC)-g-PEG-DTPA ( $1 \mathrm{~g}, 0.1 \mathrm{mmol})$ was dissolved in deionised water. To this solution, cisplatin $(0.2 \mathrm{~g}, 0.7 \mathrm{mmol})$ was added. The $\mathrm{pH}$ of the solution was adjusted to $\mathrm{pH} 7$ with $0.1 \mathrm{M} \mathrm{NaOH}$, and stirred for $72 \mathrm{~h}$. The resulted solution was dialyzed against water for five times (MWCO: 2000) to remove unbound cisplatin, and then freeze drying to afford an orange solid. The final concentration of platinum was measured by ICP-MS.

\section{Preparation of FITC labelled poly(AMPD-BAC)-g-PEG- DTPA-Pt}

FITC is attached to poly(AMPD-BAC)-g-PEG-DTPA via the reaction among isothiocyanate groups of FITC and the residual amines on poly(AMPD-BAC)-g-PEG-DTPA. In a typical process, 0.1 $\mathrm{g}$ of poly(AMPD-BAC)-g-PEG-DTPA was dissolved in $4 \mathrm{~mL}$ of dried DMSO. Then a solution of $70 \mathrm{mg}$ of FITC in $2 \mathrm{~mL}$ of DMSO was added dropwise. The reaction was performed in dark at ambient temperature overnight. The solution was dialyzed in methanol for five times.

\section{Platinum in-vitro release study}

Poly(AMPD-BAC)-g-PEG-DTPA-Pt prepared was transferred into a dialysis bag (MWCO:2000). After that, the dialysis bag was placed into a conical flask containing $40 \mathrm{~mL}$ of phosphate buffered saline and $150 \mathrm{mM} \mathrm{NaCl}$. The conical flask was kept in $37.4^{\circ} \mathrm{C}$ water bath and was being stirred at $100 \mathrm{rpm}$ throughout the release study. The release was sampled at a defined time period, and measured by ICP-MS.

\section{Cytotoxicity assay}

MDA-MB-231 cells were cultured in DMEM supplemented with $10 \% \mathrm{FBS}$ at $37^{\circ} \mathrm{C}, 10 \% \mathrm{CO}_{2}$, and $95 \%$ relative humidity. For cell viability assay, polymer solutions were prepared in serum supplemented tissue culture medium. The cells $(10,000$ cells/well) were seeded into 96well microtiter plates (Nunc, Wiesbaden, Germany). After overnight incubation, the culture medium was replaced with $100 \mu \mathrm{L}$ of serial dilutions of the polymers, and the cells were incubated for 24 and 48 hours. $20 \mu \mathrm{L}$ of sterile filtered MTT (3-(4,5-dimethylthiazol-2-yl)-2,5diphenyl tetrazolium bromide) $\left(5 \mathrm{mg} \mathrm{mL}^{-1}\right)$ stock solution in phosphate buffered saline (PBS) was added to each well. After 4 hours, unreacted dye was removed by aspiration. The formazan crystals were dissolved in $100 \mu \mathrm{L} /$ well DMSO (BDH laboratory Supplies, England) and measured spectrophotometrically in an ELISA reader (Model 550, Bio-Rad) at a 
wavelength of $570 \mathrm{~nm}$. The spectrophotometer was calibrated to zero absorbance using culture medium without cells. The relative cell growth (\%) related to control cells containing cell culture medium without polymer was calculated by $[\mathrm{A}]$ test $/[\mathrm{A}]$ control $\times 100 \%$. All the tests were performed triplicate.

\section{Fluoresence labeling}

Cells were grown on coverslips (60\% confluence), incubated with solution of FITC labeled poly(AMPD-BAC)-g-PEG-DTPA-Pt for $24 \mathrm{~h}$, washed with $1 \times$ PBS $(3 \times 2 \mathrm{ml})$, fixed with $5 \%$ formaldehyde in PBS for $20 \mathrm{~min}$ at room temperature. They were then permeabilized with $0.1 \%$ Triton X-10 in PBS (USB Corp). The sample was then washed with $1 \mathrm{x}$ PBS $(3 \times 3 \mathrm{ml})$, mounted with $200 \mu \mathrm{l}$ DAPI containing ProLong Gold antifade reagent (Invitrogen). Laser confocal fluorescence micrographs were obtained on Leica TCS SP5X. For DAPI imaging, the emission was observed at $421 \mathrm{~nm}$ with an excitation at $401 \mathrm{~nm}$; and for FITC imaging, the emission was observed at $517 \mathrm{~nm}$ with an excitation 495 nm.

\section{Dark field confocal photoacoustic microscopy system}

Our 50-MHz dark-field confocal PAM system for imaging vascular in the rat brain is below consisting of laser pulse generation and delivery, PA signal reception, and image reconstruction and display. Laser pulses, $4 \mathrm{~ns}$ wide, were generated at a frequency of $10 \mathrm{~Hz}$ by using an optical parametric oscillator (Surlite OPO Plus, Continuum, USA). The laser was pumped by a frequency-tripled Nd:YAG Q-switched laser (Surlite II-10, Continuum, USA). At the selected wavelengths, the designed contrast agent is a dominant optical absorber, producing strong optical absorption and thus guaranteeing that the detected PA signals mainly come from contrast agent $[2,34]$. The acquired PA signal at $\lambda_{410 \mathrm{~nm}}$ is sensitive to changes in contrast agent. The $50-\mathrm{MHz}$ ultrasonic transducer used in the current PAM system was custom-made by the Acoustic Sensor Co., Ltd at Taiwan. It has a $-6 \mathrm{~dB}$ fractional bandwidth of $57.5 \%$, a focal length of $9 \mathrm{~mm}$ and a $6 \mathrm{~mm}$ active element, offering an axial resolution of $32 \mu \mathrm{m}$ and a lateral resolution of $61 \mu \mathrm{m}$.

Laser energy was delivered using a 1-mm multimodal fiber. The fiber tip was coaxially aligned with a convex lens, an axicon, a plexiglass mirror, and an ultrasonic transducer on an optical bench, forming darkfield illumination that was confocal with the focal point of the ultrasonic transducer. The incident energy density on the sample surface was well within American National Standards institute (ANSI) safety limits. The transducer was immersed in an acrylic water tank during the imaging process, and the hole at the bottom of the tank was sealed with a piece of $15-\mu \mathrm{m}$ thick polyethylene film. A thin layer of ultrasonic gel was applied as a PA conductive medium, which was then attached to the thin polyethylene film to ensure reliable coupling of the PA waves with the water tank. The PA signals received by the ultrasonic transducer were pre-amplified by a low-noise amplifier, cascaded to an ultrasonic receiver (5073 PR, Olympus, USA) and then digitized and sampled by a computer-based 14-bit analog to digital (A/D) card (CompuScope $14220, \mathrm{GaGe}, \mathrm{USA}$ ) at a $200-\mathrm{MHz}$ sampling rate for data storage.

Fluctuations in the laser energy were monitored with a photodiode (DET36A/M, Thorlabs, USA). The recorded photodiode signals were measured prior to the experiment to compensate for PA signal variations caused by laser-energy instability. The achievable penetration depth of the current PA microscopy system was $3 \mathrm{~mm}$ with approximately $18-\mathrm{dB}$ SNR, where SNR is defined as the ratio of the signal peak value to the root-mean-square value of the noise. Three scan types can be provided by this system: A-line (i.e., one-dimensional images where the axis represents the imaging depth), B-scan (i.e., two-dimensional images where one axis is the lateral scanning distance and the other is the imaging depth), and C-scan (i.e., projection images from the threedimensional images) [35]. The amplitude of the envelope-detected PA signals was used in the subsequent functional imaging analysis [34].

For in vitro testing the PA contrast of the designed agent, polyethylene tubing $(\sim 20 \mathrm{~cm})$ was filled with various designed samples. Afterwards, the tubing was positioned at a depth of the transducer's focus, i.e., the depth of $9 \mathrm{~mm}$ with respect to the transducer in water tank. The system was maintained in a $25^{\circ} \mathrm{C}$ water bath throughout the experiment. The contrast changes of the developed probes were imaged using the designed PA microscopy system with $32 \times 61-\mu \mathrm{m}$ resolution. The scanning step size was $20 \mu \mathrm{m}$ for each B-scan of in vivo and in vitro experiments.

\section{Experimental animals}

Six male Wistar rats (NUS-CARE, Singapore), weighing 250 - 300 grams, were used. The animals were housed at a constant temperature and humidity with free access to food and water. Before imaging experiments, the rats fasted for 24 hours but were given water ad libitum. All animal experiments were conducted in accordance with guidelines from Institutional Animal Care and Use Committee (IACUC) at the National University of Singapore.

Rats remained anesthetized with isoflurane $2-3 \%$ in $100 \% \mathrm{O}_{2}$ and were mounted on a dorsal position over a custom-made acrylic stereotaxic holder. Anesthetized rats were mounted on the custommade acrylic stereotaxic head holder and the skin and muscle were cut away from the skull to expose the bregma landmark. The anteroposterior (AP) distance between the bregma and the interaural line was directly surveyed [36]. The bregma was $9.3 \pm 0.12 \mathrm{~mm}$ (mean \pm standard deviation [SD]) anterior to the interaural line [37]. Furthermore, a craniotomy was performed for each animal, and a bilateral cranial window of approximately 4 (horizontal) $\times 3$ (vertical) $\mathrm{mm}$ size was made with a high-speed drill. After the rat was secured to the stereotaxic frame and placed on the bed pallet, the pallet was moved to position at the bregma, which was $9 \mathrm{~mm}$ anterior to an imaginary line drawn between the centers of each ear bar (the interaural line) [34]. The interaural and bregma references were then used to position the heads in the PAM system, without additional surgery, in the following experiments.

\section{Results and Discussion}

The synthesis of biodegradable polymeric nanoparticle tethered platinum (II) is depicted in Figure 1. The Michael-addition polymerization of diamine of 4 -aminomethyl piperidine (AMPD) with an equimolar bisacrylamide (BAC) was performed in methanol. The topology of polymers can be simply tuned by varying the molar ratio of diamine to bisacrylamide. Equimolar ratio of disulfide-based bisacrylamide and diamine in Michael-addition polymerization leads to linear reduction-responsive poly(amido-amine) containing secondary and tertiary amines [38]. The disulfide bonds in poly (AMPD-BAC) backbone are sensitive to reducing agents. It may thus be expected that they would be easily cleaved into small molecules in the presence of stimuli. The gel permeation chromatography (GPC) measurements confirmed the cleavage of disulfide bonds of poly(AMPD-BAC) after incubation with GSH to yield lower molecular species as shown in Figure 2a. The peak intensity of small molecules around 22 min of elution time increased together with the decrease of peak intensity of polymer fraction with high molecular weight (around $17 \mathrm{~min}$ ), indicating that the polymer degraded into smaller molecules. 


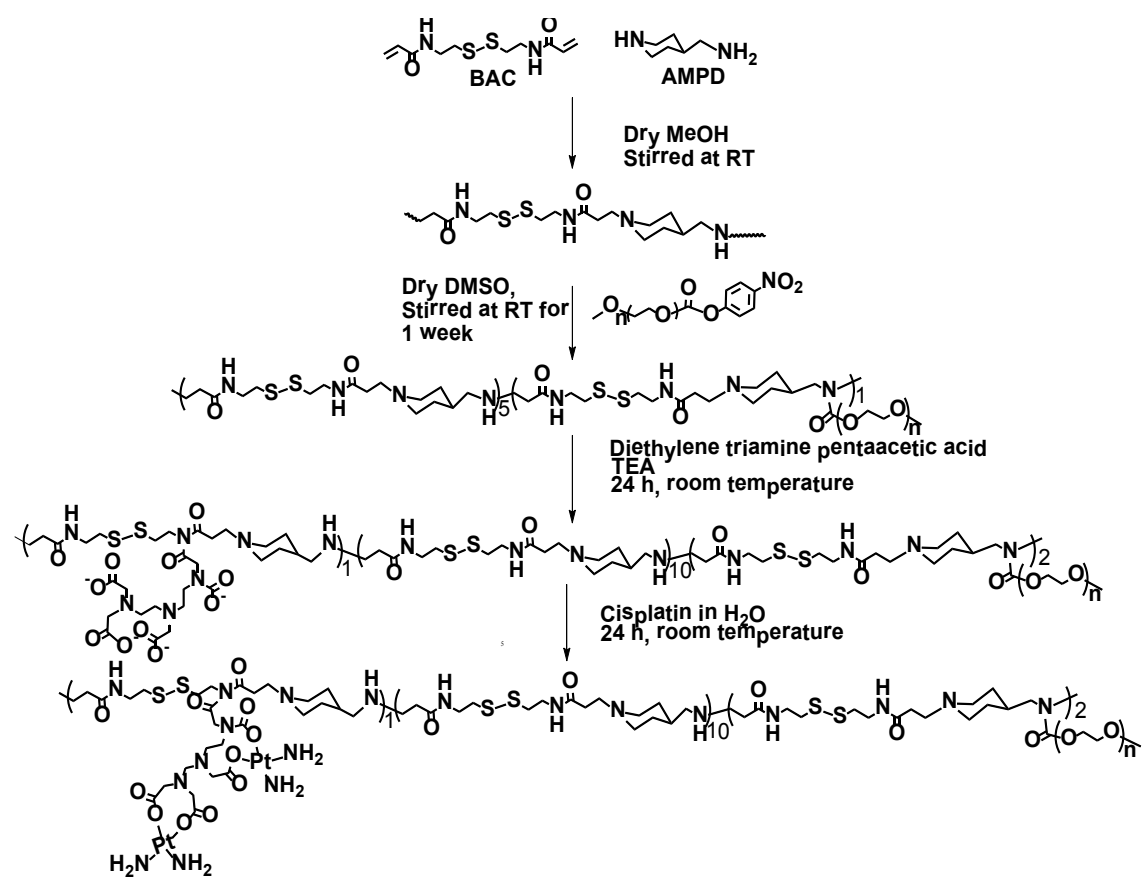

Figure 1: Synthesis pathway to platinum II-based biodegradable polymeric nanoparticle. Poly(AMPD-CAB) having numerous secondary amine group in backbone offers multiple sites for the attachment of various property modifiers. DTPA was attached to poly(AMPD-CAB) by amidation reaction of secondary amine for complexation of cisplatin. The attachments of PEG was achieved by reaction of secondary amines of poly(AMPD-CAB) with 4-nitrophenyl carbonate activated PEG.

The spherical morphology of nanoparticle of poly(AMPD-BAC)-gPEG was affected by incorporation of DTPA due to the increase of hydrophilicity of polymer (Figure 2d). Spherical nanoparticle was able to reform after complexing with cisplatin, in which the carboxylic acid binds to cisplatin, in turn reducing the hydrophilicity of polymer (Figure 2e).

Vast literature on PEG for drug delivery shows that PEG stealth layers can significantly reduce non-specific adsorption of proteins, enabling longer in vivo circulation duration, which in turn improve delivery efficacy [39-41]. The secondary amines readily react with 4-nitrophenyl carbonate activated PEG to form PEG-grafted poly(AMPD-BAC). The AMPD-BAC unit number per PEG was five as determined by ${ }^{1} \mathrm{H}-\mathrm{NMR}$ measurement. Diethylene triamine pentaacetic acid (DTPA) was incorporated through formation of amide bond between carboxylic acid group and the remaining secondary amine of poly (AMPD-BAC)-g-PEG backbone. It is used as a chelate for cisplatin to form a carboxylate platinum complex in which two chloride ligands of cisplatin are displaced by carboxylate ligands [4245]. The efficiency of incorporation of cisplatin into DTPA unit was found to be approximately $76 \%(\mathrm{w} / \mathrm{w})$ as determined by ICP-MS. After complexation of platinum complex into the poly(AMPD-BAC)g-PEG-DTPA, a yellow powder was obtained which provides a first evidence of cisplatin to complex to poly(AMPD-BAC)-g-PEG-DTPA (Figure 2f). The complexation was also confirmed by the zeta-potential measurement. The zeta potential of poly(AMPD-BAC)-g-PEG-DTPA was $-33.7 \mathrm{mV}$ and poly(AMPD-BAC)-g-PEG-DTPA-Pt was $-2.45 \mathrm{mV}$. The reduction in negative charge indicated that the carboxylic acid group was complexed with cisplatin.

As determined by dynamic light scattering (DLS), poly(AMPDBAC)-g-PEG-DTPA-Pt forms a nanoparticle with an average diameter of $118 \mathrm{~nm}$ in aqueous solution with an increase in size of poly(AMPD-
BAC)-g-PEG (average diameter: $97 \mathrm{~nm}$ ) (Figure 2c). Poly(AMPDBAC)-g-PEG-DTPA-Pt has an ideal particles size for blood vascular imaging as the optimum nanoparticle size to gain maximum blood circulation time is around $80-150 \mathrm{~nm}$ [46]. The morphology of nanoparticle was visualized by transmission electron microscopy (TEM). Unlike poly(AMPD-BAC)-g-PEG, non-spherical micelles were first obtained for poly(AMPD-BAC)-g-PEG-DTPA (Figure 2c-e) due to the increase in hydrophilicity of polymer by DTPA. As indicated by the TEM images in Figure 2e, spherical nanoparticle was able to reversibly form after complexation of platinum complex due to the binding of cisplatin to carboxylic acid group of DTPA, and thus the hydrophilicity of polymer was adjusted to form spherical nanoparticle upon complexation of platinum complex [47]. The smaller sized nanoparticle of poly(AMPD-BAC)-g-PEG-DTPA-Pt observed by TEM as compared to that measured by DLS can be attributed to the evaporation shrinkage of the PEG shell [48].

The stability of carboxylate platinum complex in nanoparticle was subsequently evaluated. The obtained release profile of cisplatin exhibited a slow release of platinum complex, at a similar rate in water, with $<5 \%$ of platinum complex content released after $60 \mathrm{~h}$ in chloride ion media, and poly(AMPD-BAC)-g-PEG-DTPA-Pt remained yellow in colour, indicating that majority of the platinum complex remained bound with polymer (Figure $3 \mathrm{a}$ ). These results are mostly attributable to the chelate in which DTPA is an excellent chelating agent and thus is expected to form a relatively stable carboxylate platinum complex. For this nanoparticle to be used as a contrast agent, its cytotoxicity was evaluated. Both toxicity results of poly(AMPD-BAC)-g-PEG-DTPA-Pt and poly(AMPD-BAC)-g-PEG nanoparticle against on normal breast cell line (MCF-10A) were similar, suggesting that the cytotoxicity of poly(AMPD-BAC)-g-PEG-DTPA-Pt is not significant (Figure $3 \mathrm{~b}$ ). These results were also reflected in the morphology of cells (Figure $3 \mathrm{c}$ - 
Citation: Kong KV, Liao LD, Goh D, Thakor NV, Olivo M (2014) Novel Biodegradable Polymer Tethered Platinum (II) for Photoacoustic Imaging. J Nanomed Nanotechnol 5: 223. doi: 10.4172/2157-7439.1000223

a

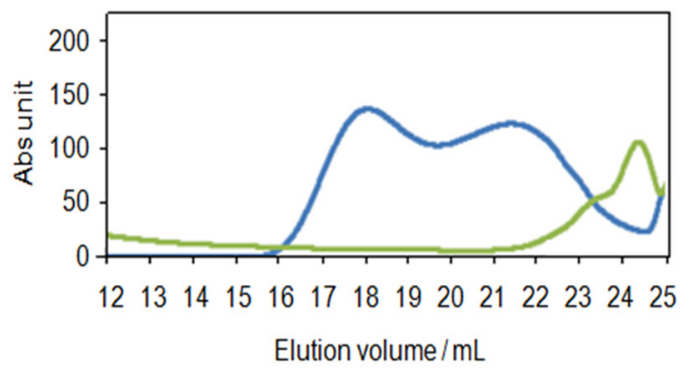

$\mathrm{b}$

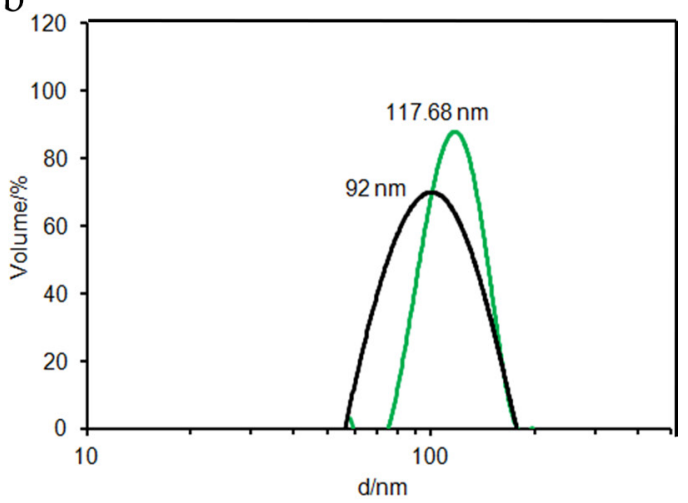

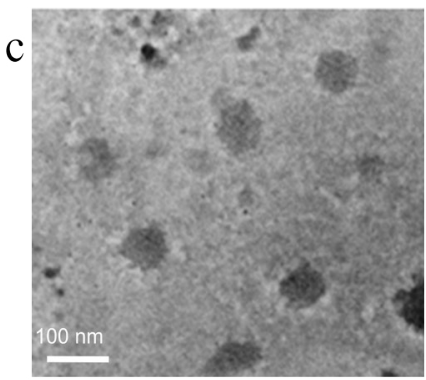
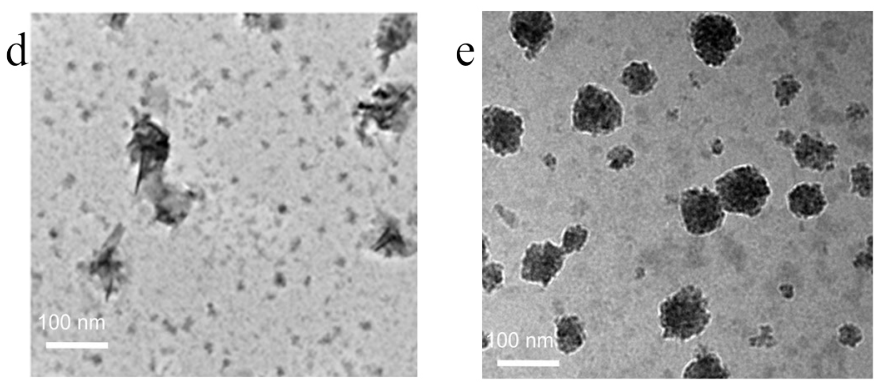

f

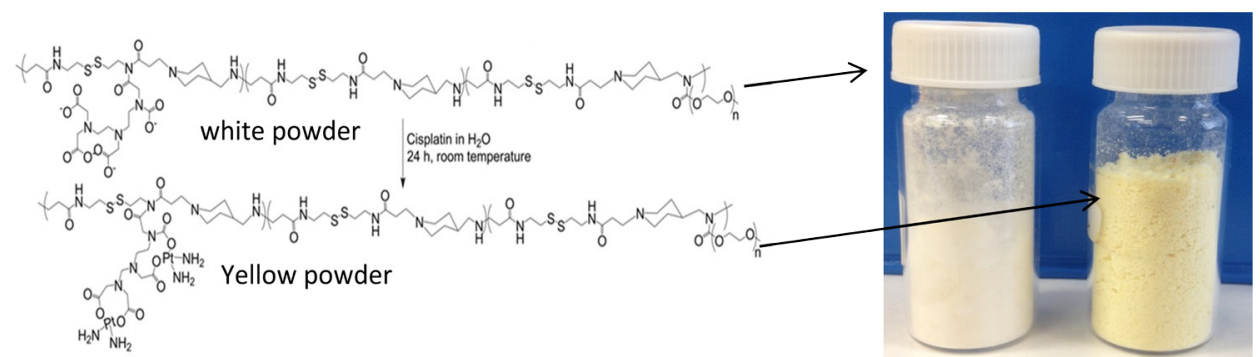

Figure 2: Characterization of nanoparticles (a) The molecular weight distribution of - poly(AMPD-BAC) and - poly(AMPD-BAC) in the incubation with $10 \mathrm{mM}$ GSH. (b) Particle size of nanoparticle as monitored by DLS. poly(AMPD-BAC)-g-PEG, poly(AMPD-BAC)-g-PEG-DTPA-Pt. The incorporation of the DTPA-Pt has effect on nanoparticle size, in which poly(AMPD-BAC)-g-PEG-DTPA-Pt forms nanoparticle (117.68 nm) with an increase in size of poly(AMPDBAC)-g-PEG (92 nm). TEM image of (c) poly(AMPD-BAC)-g-PEG, (d) poly(AMPD-BAC)-g-PEG-DTPA, (e) poly(AMPD-BAC)-g-PEG-DTPA- Pt. (f) Schematics representation of poly(AMPD-BAC)-g-PEG-DTPA after incorporation of Pt.

e). The great stability of platinum complex in nanoparticle clearly rules out any cytotoxic effect from platinum complex.

The PA measurement was performed in the visible region. This allows high resolution images to be obtained, and are sufficient for superficial brain vasculature imaging. The generated photoacoustic signal is due to electronic transitions in the central platinum metal which was assigned to $\mathrm{d}$-d transitions. The results show that poly(AMPD-BAC)-g-PEG-DTPA-Pt can provide high PA signal in which PA of poly(AMPD-BAC)-g-PEG-DTPA-Pt is 3.7 times higher than water (Figure 4). The investigated concentration dependence of poly(AMPD-BAC)-g-PEG-DTPA-Pt, PA signals have been measured with a series of poly(AMPD-BAC)-g-PEG-DTPA-Pt samples ranging from 1.2 to $15 \mathrm{mg} / \mathrm{mL}$ and the results are shown in Figure 4 . A linear response is shown for different concentrations samples.

To further demonstrate the value of poly(AMPD-BAC)-g-PEGDTPA-Pt as a PA contrast agent, we incubated MCF-10A cells with the contrast agent and removed excess contrast agents prior to PA imaging. As shown in Figure 5, there are no intrinsic PA signals pre-incubation, but after incubation with poly(AMPD-BAC)-g-PEG-DTPA-Pt (Figure $5 b$ ), clear PA C-scan images of MCF-10A can be observed as shown in Figure $5 \mathrm{c}$. The interaction of poly(AMPD-BAC)-g-PEG-DTPA-Pt with cells was further confirmed through visualization via fluorescence confocal microscopy using FITC-labelled poly(AMPD-BAC)-g-PEGDTPA-Pt. The results in Figure 5d show that MCF-10A cells showed strong fluorescence, suggesting that poly(AMPD-BAC)-g-PEG-DTPAPt can be easily transported into cells. It also provides a clear indication that poly(AMPD-BAC)-g-PEG-DTPA-Pt is distributed in cytoplasm and nuclear region. In order to validate the possibility of internalization of poly(AMPD-BAC)-g-PEG-DTPA-Pt into nucleus over envelopment, depth profiling experiment was conducted. Figure $5 \mathrm{e}$ shows that the poly(AMPD-BAC)-g-PEG-DTPA-Pt has penetrated into the nucleus with uneven distribution. All images were taken from a stack of slices scanned through the cells with a full scanning depth of approximately 
Citation: Kong KV, Liao LD, Goh D, Thakor NV, Olivo M (2014) Novel Biodegradable Polymer Tethered Platinum (II) for Photoacoustic Imaging. J Nanomed Nanotechnol 5: 223. doi: 10.4172/2157-7439.1000223

$\mathrm{a}$

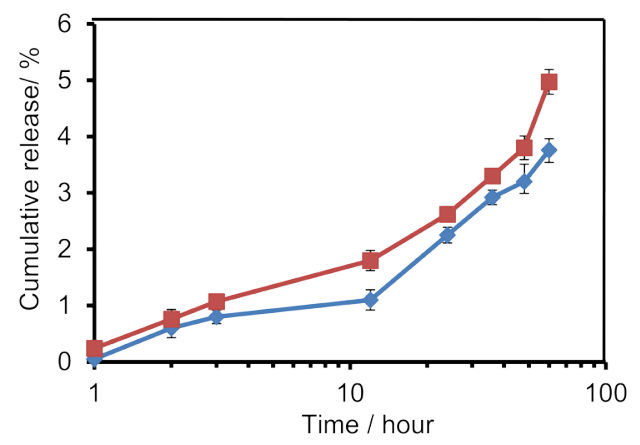

b

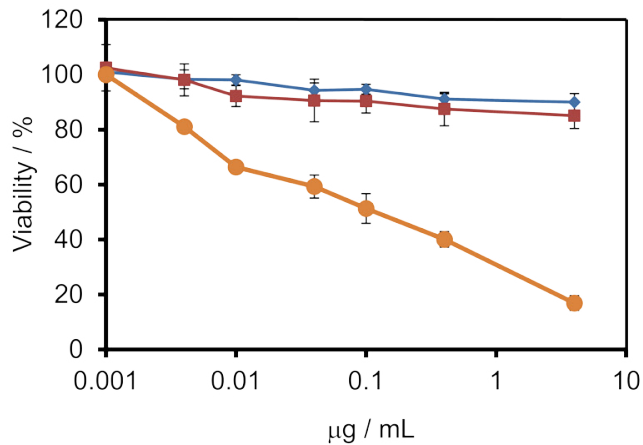

$\mathrm{c}$

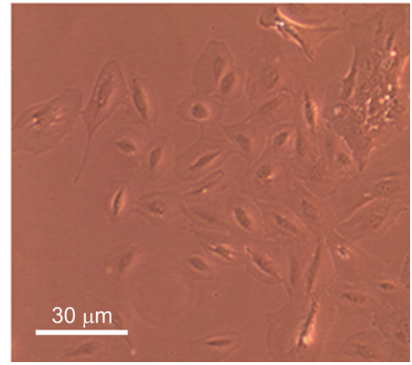

d

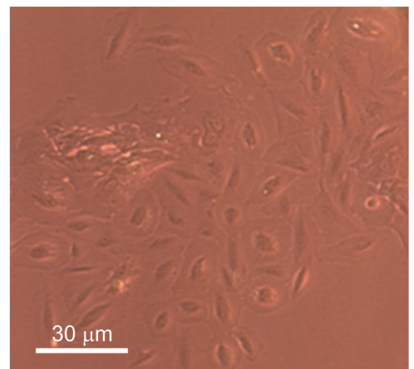

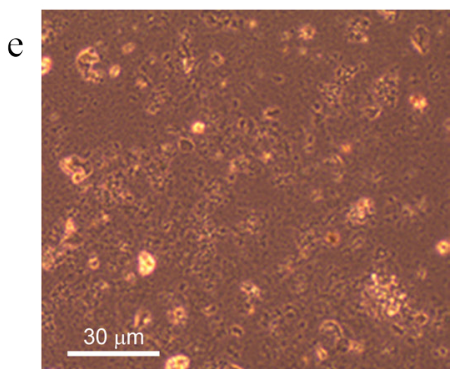

Figure 3: ((a) Cumulative release of poly(AMPD-BAC)-g-PEG-DTPA-Pt in water ( - - ) and in chloride solution (150 mM NaCl) $(-4)$. There is no significant release of platinum complex in either water or high chloride solution which is mainly attributed to the strong chelation of DTPA. (b) Effects of - - poly(AMPD-BAC)g-PEG, - poly(AMPD-BAC)-g-PEG-DTPA-Pt and - - cisplatin on viability of MCF-10A after $24 \mathrm{~h}$ incubation. Poly(AMPD-BAC)-g-PEG-DTPA-Pt shows little cytotoxicity to cells as poly(AMPD-BAC)-g-PEG. Cytotoxicity profile of cisplatin is presented for comparison. Optical images of MCF-10A cells $24 \mathrm{~h}$ incubation with $10 \mathrm{mg} / \mathrm{mL}$ concentrations of (c) Poly(AMPD-BAC)-g-PEG, (d) Poly(AMPD-BAC)-g-PEG-DTPA-Pt and (e) cisplatin.
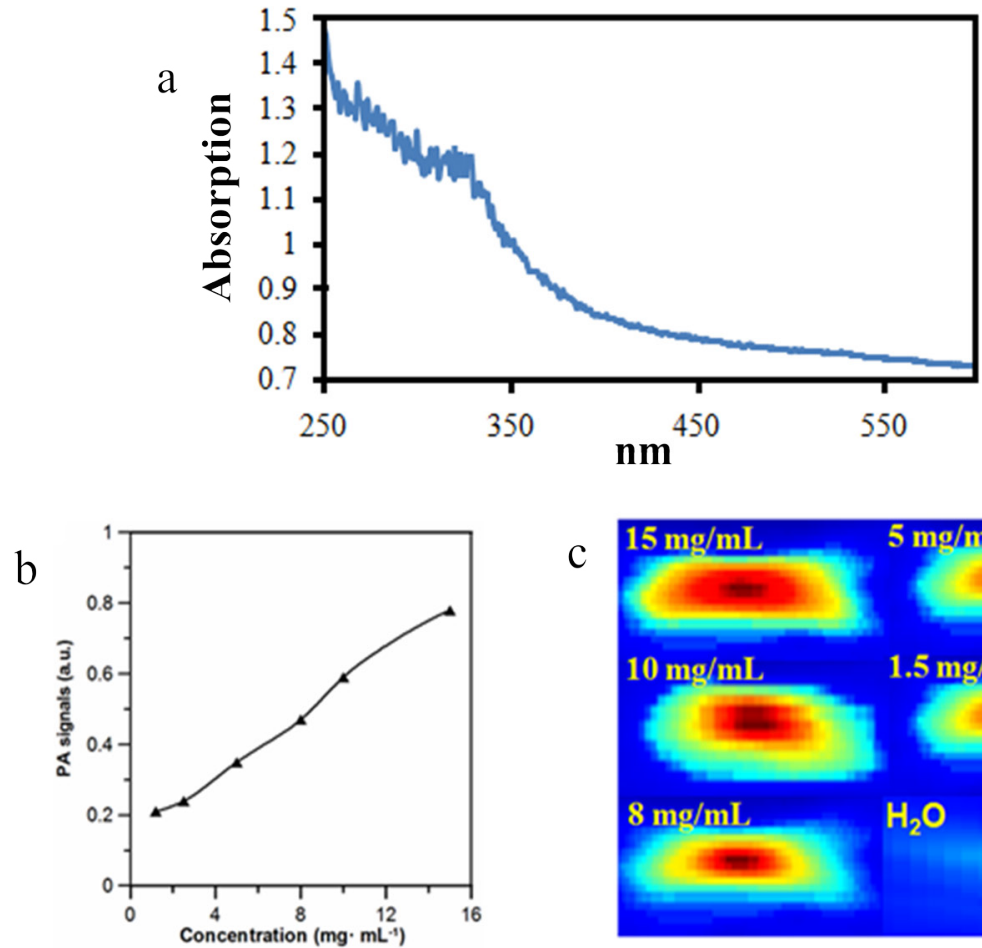

C

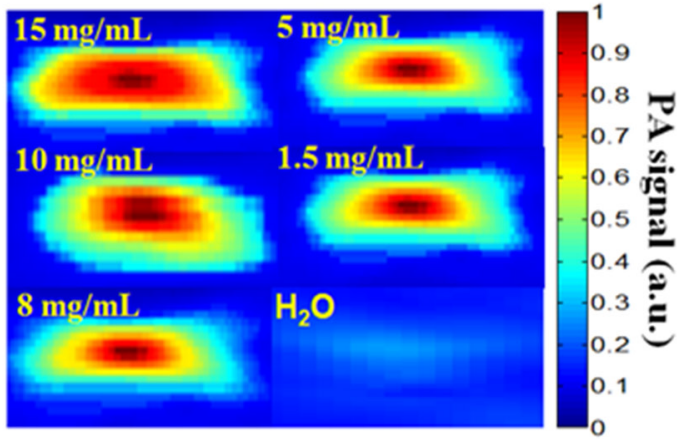

Figure 4: (a) UV-vis spectrum of poly(AMPD-BAC)-g-PEG-DTPA-Pt. (b) PA signals of a series of different concentration of poly(AMPD-BAC)-g-PEG-DTPA-Pt and water. (c) False color images representing the relative PA signal strengths PA B-scan. 
Citation: Kong KV, Liao LD, Goh D, Thakor NV, Olivo M (2014) Novel Biodegradable Polymer Tethered Platinum (II) for Photoacoustic Imaging. J Nanomed Nanotechnol 5: 223. doi: 10.4172/2157-7439.1000223

a

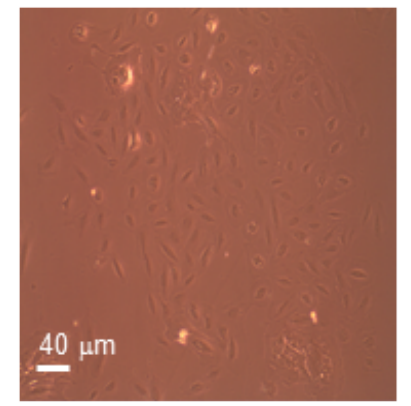

C

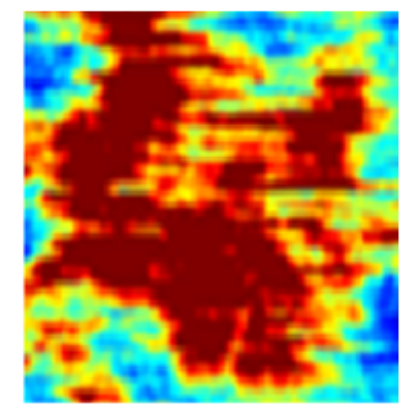

e
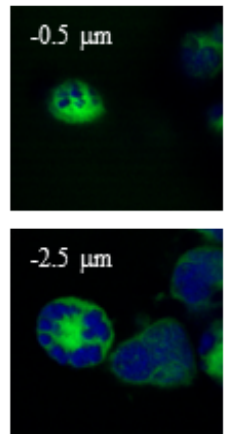
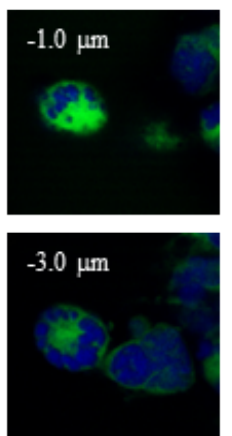

$\mathrm{b}$

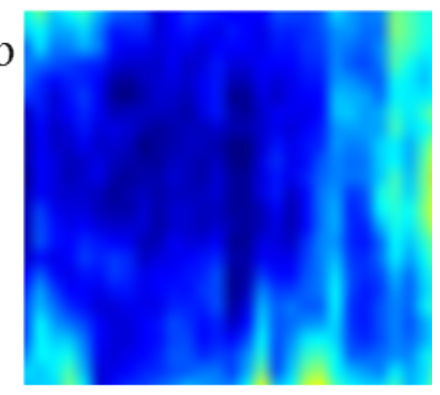

$\mathrm{d}$
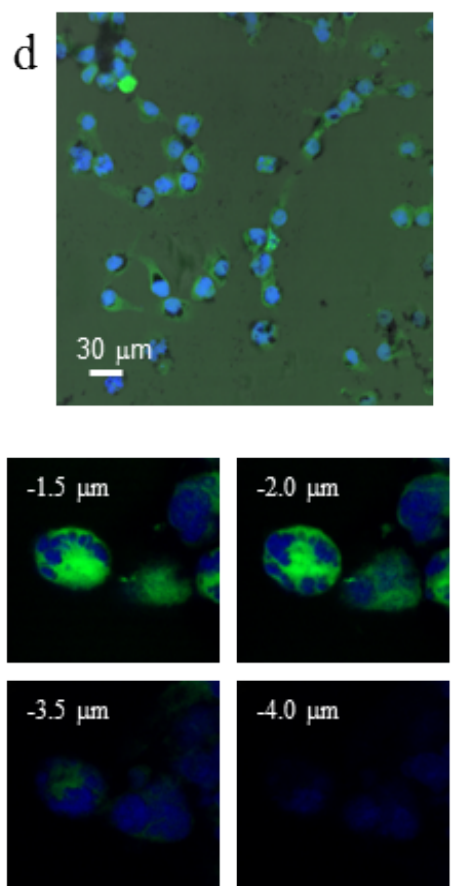

$-4.0 \mu \mathrm{m}$

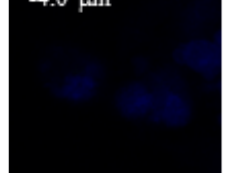

Figure 5: (a) Optical images of MCF-10A cells. PA C-scan on MCF-10A (b) before and (c) after incubation with poly(AMPD-BAC)-g-PEG-DTPA-Pt. (d) Fluorescence images of MCF-10A cells incubated with FITC (green) labeled of poly(AMPD-BAC)-g-PEG-DTPA-Pt. Cell nucleus was stained with DAPI (blue). (e) Confocal fluorescence images of MCF-10A cells incubated with poly(AMPD-BAC)-g-PEG-DTPA-Pt for $24 \mathrm{~h}$. Images were acquired at different depths of $z$ axis (0.5 $\mu$ m).

$5 \mu \mathrm{m}$ and a scanning step of $0.5 \mu \mathrm{m}$. The observation suggests that poly(AMPD-BAC)-g-PEG-DTPA-Pt entered the cell and nucleus.

The application of poly(AMPD-BAC)-g-PEG-DTPA-Pt was further demonstrated in animal. The PA measurement was performed in the visible region. This allows high resolution images to be obtained, and are sufficient for superficial brain vasculature imaging. The generated photoacoustic signal is due to electronic transitions in the central platinum metal which was assigned to $d$ - $d$ transitions. The openskull window of the rat cortical surface was shown in Figure 6b. The superior sagittal sinus (SSS) and other vessels on the cortical surface could be observed visually. The SSS is the largest vein in the rodent brain cortex Imaging of cerebral venous especially SSS is important for traumatic brain injury study [49]. Although SSS could be imaged by PA microscopy, the detected PA signals from the SSS were weaker than those from other cortical vessels. Similar limitations have been also reported by other research groups $[34,50]$. We envisage that photoacaoustic contrast agent may significantly enhance the inherently poor image contrast of SSS. The potential of enhancing SSS detection is to provide a means to study SSS-related cerebral issues. As shown in Figure 6, the rat cerebral cortex was imaged by custom-designed
PAM [34,51] before and after a single injection of poly(AMPD-BAC)g-PEG-DTPA-Pt $(5 \mathrm{mg} / \mathrm{mL})$. Compared to the brain image based on the intrinsic optical contrast (Figure 6c), the image acquired $20 \mathrm{~min}$ after the administration of poly(AMPD-BAC)-g-PEG-DTPA-Pt shows the brain vasculature with greater clarity, especially the SSS (Figure 6c), suggesting the strong ability of poly(AMPD-BAC)-g-PEG-DTPA-Pt to generate PA signal in vivo for PA imaging of brain vessels of live rat as well as a low brain parenchymal background. It is known that polymeric agents pose significantly prolonged and enhanced blood pool imaging. The absorption signals from the PAM images acquired at each time point after injection of poly(AMPD-BAC)-g-PEG-DTPA-Pt were integrated and normalized to the signal integration of the pre-injection image. As shown in Figure 6d, the highest PA signal was acquired at $20 \mathrm{~min}$ after a single injection of the contrast agent $(5 \mathrm{mg} / \mathrm{mL})$ and the intensity was about $34 \%$ higher than that of the pre-injection value. The absorption enhancement remained high, even after $60 \mathrm{~min}$, with an average $23 \%$ increase over the pre-injection value which indicates a sufficient amount of poly(AMPD-BAC)-g-PEG-DTPA-Pt circulating in the blood.

The degradation process of disulfide group is relatively fast and 
Citation: Kong KV, Liao LD, Goh D, Thakor NV, Olivo M (2014) Novel Biodegradable Polymer Tethered Platinum (II) for Photoacoustic Imaging. J Nanomed Nanotechnol 5: 223. doi: 10.4172/2157-7439.1000223
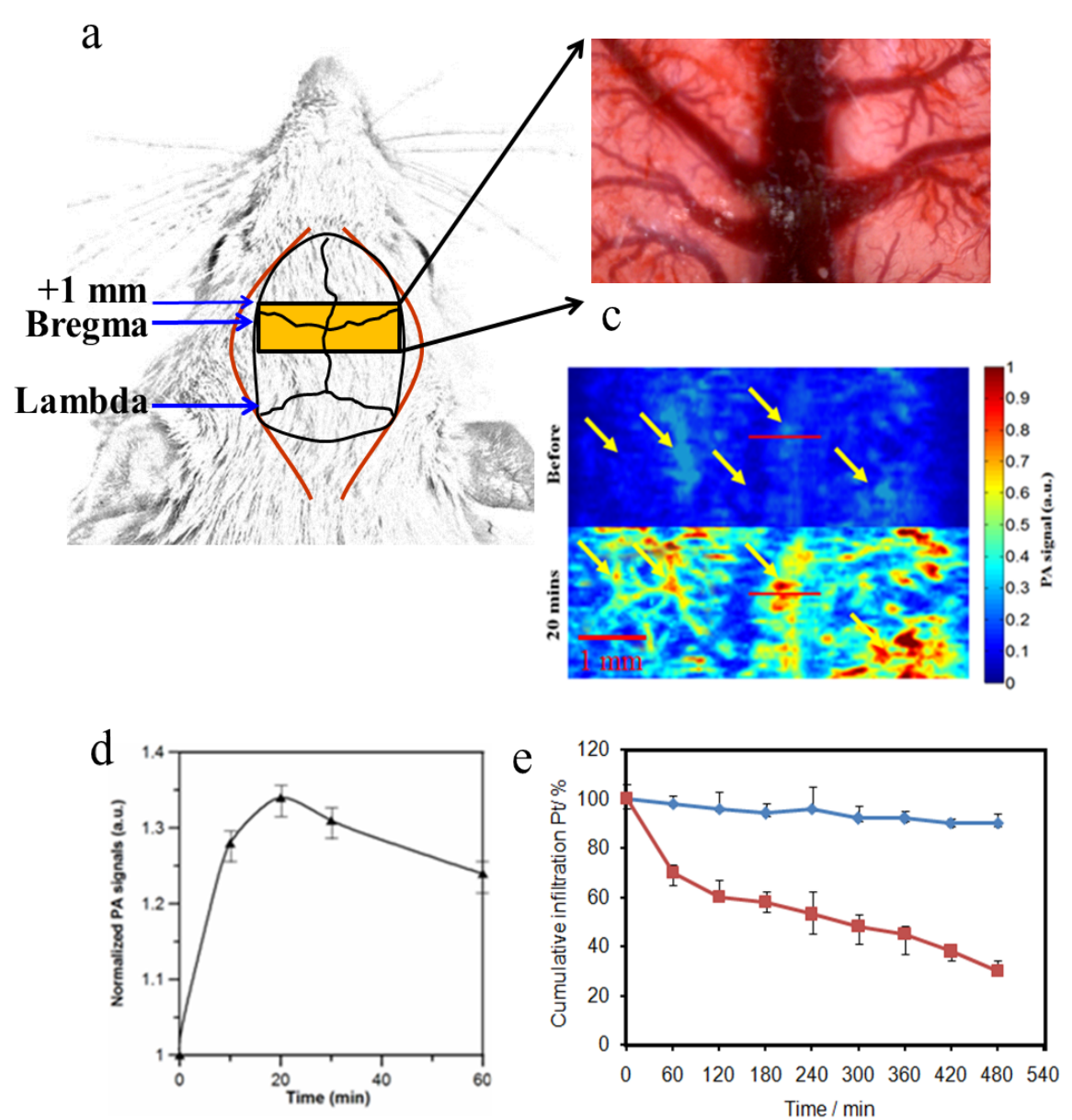

Figure 6: PAM imaging superficial brain vasculature of a rat employing poly(AMPD-BAC)-g-PEG-DTPA-Pt and light at $410 \mathrm{~nm}$. (a) The yellow area indicates the selected imaging region. (b) Photograph of the superficial rat cortical blood vessels, with the SSS indicated. (c) PA image acquired before and 20 min after the intravenous injection of poly(AMPD-BAC)-g-PEG-DTPA-Pt. (d) The integrated absorption calculated from the in vivo brain images of six rats at different times following the injection of poly(AMPD-BAC)-g-PEG-DTPA-Pt. The presented values were normalized to that of the integrated absorption of the image obtained before the injection. (e) In vitro degradation of poly(AMPD-BAC)-g-PEG-DTPA-Pt in a thiols solution mimicking the human plasma thiol composition. - Tris buffer $\mathrm{pH} 7.4$ and - solution mimicking human plasma free thiols.

can happen within minutes to hours [52]. This is significantly faster than the degradation kinetics of other functionalities such as esters and carbonates, in which degradation of both functional groups require days to weeks. The degradability of poly(AMPD-BAC)-g-PEG-DTPAPt was evaluated in which poly(AMPD-BAC)-g-PEG-DTPA-Pt was incubated in the thiol solutions mimicking the plasma composition of the endogenous thiols. Samples were collected at different time points and ultrafiltrated with a filter with a molecular weight cutoff 2000 $\mathrm{Da}$. The percentage of unfiltrated Pt was determined by ICP-MS and calculated to represent the relative degradability of poly(AMPD-BAC)g-PEG-DTPA-Pt. As shown in Figure 6e, poly(AMPD-BAC)-g-PEGDTPA-Pt degraded rapidly during 8 hours incubation, whereas little degradation was determined in thiol-free solution. Taken together, the poly(AMPD-BAC)-g-PEG-DTPA-Pt with multiple disulfide bonds resulted in strong contrast enhancement in the blood vessel. The disulfide bonds facilitated the excretion of platinum chelate.

\section{Conclusions}

We have demonstrated, for the first time, a biodegradable PA contrast agent with the combination of platinum complex and biodegradable polymer. The property of biodegradation and detectable
PA signal offers a versatile approach for brain vascular imaging which can easily be extended to other transition metal complex and biodegradable polymer due to the availability of multiple sites for the attachment of various ligands as property modifiers. The advantages of using biodegradable polymer are its intriguing properties such as longevity, intracellular penetration and versatility of incorporating transition metal complexes. Given that, it can be a useful platform to develop PA contrast agent catered to the specific requirements of preclinical and clinical applications.

\section{Acknowledgement}

This work was supported by the SBIC (Biomedical Research Council, Agency for Science, Technology and Reseach, Singapore).

\section{Appendix}

\section{Supplementary Information}

Supplementary data associated with this article can be found in the online version.

\section{References}

1. Plummer R, Wilson RH, Calvert H, Boddy AV, Griffin M, et al. (2011) A Phase clinical study of cisplatin-incorporated polymeric micelles (NC-6004) in patients with solid tumours. Br J Cancer 104: 593-598. 
Citation: Kong KV, Liao LD, Goh D, Thakor NV, Olivo M (2014) Novel Biodegradable Polymer Tethered Platinum (II) for Photoacoustic Imaging. J Nanomed Nanotechnol 5: 223. doi: 10.4172/2157-7439.1000223

2. Liao LD, Lin CT, Shih YY, Lai HY, Zhao WT, et al. (2012) Investigation of the cerebral hemodynamic response function in single blood vessels by functional photoacoustic microscopy. J Biomed Opt 17: 061210.

3. Liao LD, Tsytsarev V, Delgado-Martínez I, Li ML, Erzurumlu R, et al. (2013) Neurovascular coupling: in vivo optical techniques for functional brain imaging. Biomed Eng Online 12: 38.

4. Kircher MF, de la Zerda A, Jokerst JV, Zavaleta CL, Kempen PJ, et al. (2012) A brain tumor molecular imaging strategy using a new triple-modality MRIphotoacoustic-Raman nanoparticle. Nat Med 18: 829-834

5. Tsytsarev V, Bernardelli C, Maslov KI (2012) Living brain optical imaging technology, methods and applications. J Neurosci Neuroeng 1: 180-192.

6. Wang X, Ku G, Wegiel MA, Bornhop DJ, Stoica G, et al. (2004) Noninvasive photoacoustic angiography of animal brains in vivo with near-infrared light and an optical contrast agent. Opt Lett 29: 730-732.

7. Jokerst JV, Cole AJ, Van de Sompel D, Gambhir SS (2012) Gold nanorods for ovarian cancer detection with photoacoustic imaging and resection guidance via Raman imaging in living mice. ACS Nano 6: 10366-10377.

8. Pan D, Cai X, Yalaz C, Senpan A, Omanakuttan K, et al. (2012) Photoacoustic sentinel lymph node imaging with self-assembled copper neodecanoate nanoparticles. ACS Nano 6: 1260-1267.

9. Gray HB (2003) Biological inorganic chemistry at the beginning of the $21 \mathrm{st}$ century. Proc Natl Acad Sci U S A 100: 3563-3568.

10. Jaouen G (2006) Bioorganometallics: John Wiley \& Sons

11. Cooley ME, Davis LE, DeStefano M, Abrahm J (1994) Cisplatin: a clinical review. Part I--Current uses of cisplatin and administration guidelines. Cancer Nurs 17: 173-184.

12. Runge VM, Schoerner W, Niendorf HP, Laniado M, Koehler D, et al. (1985) Initial clinical evaluation of gadolinium DTPA for contrast-enhanced magnetic resonance imaging. Magn Reson Imaging 3: 27-35.

13. Chválová K, Brabec V, Kaspárková J (2007) Mechanism of the formation of DNA-protein cross-links by antitumor cisplatin. Nucleic Acids Res 35: 1812 1821.

14. Crisafuli FA, Cesconetto EC, Ramos EB, Rocha MS (2012) DNA-cisplatin interaction studied with single molecule stretching experiments. Integr Biol (Camb) 4: 568-574

15. Damsma GE, Alt A, Brueckner F, Carell T, Cramer P (2007) Mechanism of transcriptional stalling at cisplatin-damaged DNA. Nat Struct Mol Biol 14:11271133.

16. Krishna V, Singh A, Sharma P, Iwakuma N, Wang Q, et al. (2010) Polyhydroxy fullerenes for non-invasive cancer imaging and therapy. Small 6: 2236-2241.

17. Lee JU, Hosotani R, Wada M, Doi R, Kosiba T, et al. (1997) Mechanism of apoptosis induced by cisplatin and VP-16 in PANC-1 cells. Anticancer Res 17 $3445-3450$.

18. Lieberthal W, Triaca V, Levine J (1996) Mechanisms of death induced by cisplatin in proximal tubular epithelial cells: apoptosis vs. necrosis. Am J Physiol 270: F700-708.

19. Boulikas T, Vougiouka M (2004) Recent clinical trials using cisplatin, carboplatin and their combination chemotherapy drugs (review). Oncol Rep 11: 559-595.

20. Haxton KJ, Burt HM (2008) Hyperbranched polymers for controlled release of cisplatin. Dalton Trans : 5872-5875.

21. Nishiyama N, Okazaki S, Cabral H, Miyamoto M, Kato $Y$, et al. (2003) Nove cisplatin-incorporated polymeric micelles can eradicate solid tumors in mice. Cancer Res 63: 8977-8983.

22. Blacklock J, You YZ, Zhou QH, Mao G, Oupický D (2009) Gene delivery in vitro and in vivo from bioreducible multilayered polyelectrolyte films of plasmid DNA. Biomaterials 30: 939-950.

23. Hong CY, You YZ, Wu DC, Liu Y, Pan CY (2007) Thermal control over the topology of cleavable polymers: from linear to hyperbranched structures. J Am Chem Soc 129: 5354-5355.

24. Jain R, Standley SM, Frechet JMJ (2007) Synthesis and degradation of pHsensitive linear poly(amidoamine)s. Macromolecules 40: 452-457.

25. Mather BD, Viswanathan K, Miller KM, Long TE (2006) Michael addition reactions in macromolecular design for emerging technologies. Prog Polym Sci 31: 487-531.
26. Yang W, Pan CY (2009) Synthesis and Fluorescent Properties of Biodegradable Hyperbranched Poly(amido amine)s. Macromol Rapid Commun 30: 2096-2101.

27. Meng F, Hennink WE, Zhong Z (2009) Reduction-sensitive polymers and bioconjugates for biomedical applications. Biomaterials 30: 2180-2198.

28. Gao K, Huang L (2009) Nonviral methods for siRNA delivery. Mol Pharm 6 : 651-658.

29. Gao W, Chan JM, Farokhzad OC (2010) pH-Responsive nanoparticles for drug delivery. Mol Pharm 7: 1913-1920.

30. Hu Y, Litwin T, Nagaraja AR, Kwong B, Katz J, et al. (2007) Cytosolic delivery of membrane-impermeable molecules in dendritic cells using $\mathrm{pH}$-responsive core-shell nanoparticles. Nano Lett 7: 3056-3064.

31. Quu LY, Zheng C, Zhao QH (2012) Mechanisms of Drug Resistance Reversal in Dox-Resistant MCF-7 Cells by pH-Responsive Amphiphilic Polyphosphazene Containing Diisopropylamino Side Groups. Mol Pharm 9: 1109-1117.

32. Stanton MG, Colletti SL (2010) Medicinal chemistry of siRNA delivery. J Med Chem 53: 7887-7901.

33. Xiong MP, Bae Y, Fukushima S, Forrest ML, Nishiyama N, et al. (2007) pHresponsive Multi-PEGylated dual cationic nanoparticles enable charge modulations for safe gene delivery. ChemMedChem 2: 1321-1327.

34. Liao LD, Li ML, Lai HY, Shih YYI, Lo YC, et al. (2010) Imaging brain hemodynamic changes during rat forepaw electrical stimulation using functional photoacoustic microscopy. Neuroimage 52: 562-570.

35. Zhang HF, Maslov K, Wang LV (2007) In vivo imaging of subcutaneous structures using functional photoacoustic microscopy. Nat Protoc 2: 797-804

36. Paxinos G, Watson C (2007) The Rat Brain in Stereotaxic Coordinates. San Diego: Academic Press.

37. Chen YY, Shih YY, Lo YC, Lu PL, Tsang S, et al. (2010) MicroPET imaging of noxious thermal stimuli in the conscious rat brain. Somatosens Mot Res 27 69-81.

38. You YZH, Hong CY, Pan CY (2009) Facile One-Pot Approach for Preparing Dually Responsive Core Shell Nanostructure. Macromolecules 43: 573-575.

39. Antonietti M, Forster S (2003) Vesicles and liposomes: A self-assembly principle beyond lipids. Adv Mater 15: 1323-1333.

40. Discher DE, Eisenberg A (2002) Polymer vesicles. Science 297: 967-973.

41. Lee JC, Bermudez H, Discher BM, Sheehan MA, Won YY, et al. (2001) Preparation, stability, and in vitro performance of vesicles made with diblock copolymers. Biotechnol Bioeng 73: 135-145.

42. Di Pasqua AJ, Goodisman J, Kerwood DJ, Toms BB, Dubowy RL, et al. (2006) Activation of carboplatin by carbonate. Chem Res Toxicol 19: 139-149.

43. Nishiyama N, Okazaki S, Cabral H, Miyamoto M, Kato Y, et al. (2003) Nove cisplatin-incorporated polymeric micelles can eradicate solid tumors in mice. Cancer Res 63: 8977-8983.

44. Tseng CL, Su WY, Yen KC, Yang KC, Lin FH (2009) The use of biotinylatedEGF-modified gelatin nanoparticle carrier to enhance cisplatin accumulation in cancerous lungs via inhalation. Biomaterials 30: 3476-3485

45. Ye H, Jin L, Hu R, Yi Z, Li J, et al. (2006) Poly(gamma, L-glutamic acid)-cisplatin conjugate effectively inhibits human breast tumor xenografted in nude mice. Biomaterials 27: 5958-5965.

46. Brinkhuis RP, Rutjes FPJT, van Hest JCM (2011) Polymeric vesicles in biomedical applications. Polym Chem 2: 1449-1462.

47. Huynh VT, Quek JY, de Souza PL, Stenzel MH (2012) Block copolymer micelles with pendant bifunctional chelator for platinum drugs: effect of spacer length on the viability of tumor cells. Biomacromolecules 13: 1010-1023.

48. Chen W, Meng F, Li F, Ji SJ, Zhong Z (2009) pH-Responsive Biodegradable Micelles Based on Acid-Labile Polycarbonate Hydrophobe: Synthesis and Triggered Drug Release. Biomacromolecules 10: 1727-1735.

49. Petrov IY, Petrov Y, Prough DS, Deyo DJ, Cicenaite I, et al. (2012) Optoacoustic monitoring of cerebral venous blood oxygenation through extracerebral blood. Biomed Opt Express 3: 125-136.

50. Stein EW, Maslov K, Wang LV (2009) Noninvasive, in vivo imaging of bloodoxygenation dynamics within the mouse brain using photoacoustic microscopy J Biomed Opt 14: 020502. 
Citation: Kong KV, Liao LD, Goh D, Thakor NV, Olivo M (2014) Novel Biodegradable Polymer Tethered Platinum (II) for Photoacoustic Imaging. J Nanomed Nanotechnol 5: 223. doi: 10.4172/2157-7439.1000223

Page 10 of 10

51. Liao LD, Lin CT, Shih YY, Lai HY, Zhao WT, et al. (2012) Investigation of the cerebral hemodynamic response function in single blood vessels by functional photoacoustic microscopy. J Biomed Opt 17: 061210.
52. Dollendorf C, Hetzer M, Ritter H (2013) Polymeric redox-responsive delivery systems bearing ammonium salts cross-linked via disulfides. Beilstein $\mathrm{J}$ Org Chem 9: 1652-1662. 